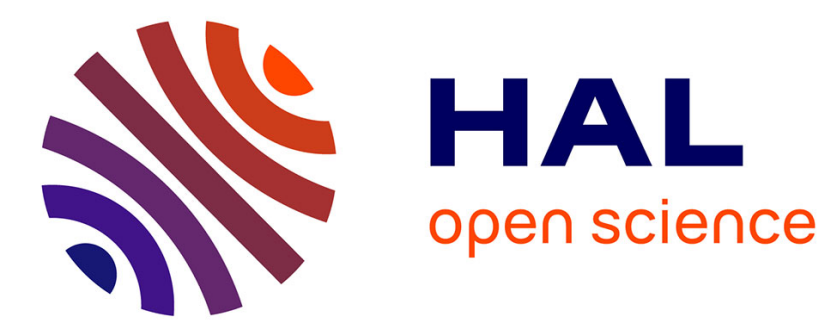

\title{
Un état des questions sur les sanctuaires et les pratiques cultuelles de la Gaule celtique
}

Patrice Arcelin, Jean-Louis Brunaux

\section{To cite this version:}

Patrice Arcelin, Jean-Louis Brunaux. Un état des questions sur les sanctuaires et les pratiques cultuelles de la Gaule celtique. Gallia - Archéologie de la France antique, 2003, Cultes et sanctuaires en France à l'âge du Fer, 60, pp.5-8. 10.3406/galia.2003.3142 hal-01911703

\section{HAL Id: hal-01911703 \\ https://hal.science/hal-01911703}

Submitted on 9 Jan 2020

HAL is a multi-disciplinary open access archive for the deposit and dissemination of scientific research documents, whether they are published or not. The documents may come from teaching and research institutions in France or abroad, or from public or private research centers.
L'archive ouverte pluridisciplinaire HAL, est destinée au dépôt et à la diffusion de documents scientifiques de niveau recherche, publiés ou non, émanant des établissements d'enseignement et de recherche français ou étrangers, des laboratoires publics ou privés.

\section{(ㅇ)(1) $\$$}

Distributed under a Creative Commons Attribution - NonCommercial - NoDerivatives| 4.0 


\title{
UN ÉTAT DES QUESTIONS \\ SUR LES SANCTUAIRES ET LES PRATIQUES CULTUELLES DE LA GAULE CELTIQUE
}

\author{
Patrice ArCELIN et Jean-Louis BrunAuX
}

\section{UN BIL $\Lambda$ N DES RECHERCHES RÉCENTES}

Les éléments réunis dans ce dossier n'ont pas l'ambition de dresser un inventaire exhaustif des lieux reconnus pour les manifestations des pratiques religieuses de la Gaule celtique. Il s'agit bien d'une autre approche, celle d'un questionnement autour des informations recueillies lors des deux dernières décennies, dans le cadre de la France contemporaine. La démarche choisie se veut foncièrement pragmatique, prenant en compte la réalité des ressources interrégionales en ce domaine. De ce fait, le découpage en cinq zones de présentation ne couvre pas l'ensemble de la Gaule antique, laissant à l'écart de vastes secteurs géographiques (fig. 1). On le regrettera, mais cette dernière réalité est également hautement significative de la pauvreté des recherches dans le champ qui nous concerne, comme de leur diffusion restreinte. On en retiendra surtout la nécessité de leur rapide développement dans un avenir que l'on espère proche. En ce qui concerne les découvertes majeures antérieures, les pages qui suivent ne les ignorent pas, les considérant surtout par une relecture à la lumière des connaissances actuelles, voire en les réinsérant dans une vision plus sociologique et enrichie.

Le découpage choisi est à l'image de la dynamique actuelle de la recherche française pour l'âge du Fer, comme de ses faiblesses. Il n'est qu'un cadre pratique de présentation des analyses et des réflexions régionales dans le domaine des cultes et de leurs sanctuaires, surtout en l'absence patente d'une homogénéisation formelle des modalités d'enquêtes et des caractères de reconnaissance des signes du sacré. En somme, cette ventilation est un état régionalisé des potentialités reconnues, des interrogations qui en découlent et des réponses provisoirement apportées dans un cadre culturel élargi. Les orientations traditionnelles de la recherche archéologique française sur les âges du Fer sont également prégnantes dans le domaine du cultuel et souvent en déterminent l'apparence des carac- tères préférentiels ou des silences. Ainsi la primautć dcs investigations sur les habitats et les nécropoles en milieu ouvert des régions septentrionale et orientale de la France trouve à l'évidence un écho dans la multiplicité des sanctuaires de plaine. A contrario, dans les régions du Sud-Est méditerranéen où l'accent a longtemps été porté sur les habitats perchés et la recherche de leurs nécropoles, les autres structures en milieu ouvert sont demeurées négligées. Dans ce premier cas, les connaissances sur les habitats groupés restent embryonnaires et le caractère jugé exceptionnel d'un village comme Acy-Romance (Ardennes) ne l'est peut-être pas tant que cela. À l'opposé, dans le Midi, en Languedoc ou près de Marseille, les recherches préventives résultant des grands travaux en plaine fournissent dorénavant les premières images d'un milieu " rural " plus complexe qu'on ne le pensait voici encore une dizaine d'années. Les enclos repérés ou fouillés à Vestric-et-Candiac, au sud de Nîmes, ouvrent ainsi une nouvelle série méridionale de structures fossoyées de plaine, de nature cultuelle, qu'elle soit funéraire ou non. Dans ces constats de répartition géographique, les spécificités environnementales sont essentielles. Les rédacteurs des différents chapitres et de leurs notices prennent en compte les contraintes du milieu au même titre que les contextes culturels pour souligner des pratiques régionales récurrentes à l'image du rôle des grottes et des sources en relief karstique ou des immersions d'offrandes en terrains humides et marécageux.

\section{LA NÉCESSITÉ DE CROISER LES SOURCES D'INFORMATION}

Les chapitres du dossier conjuguent trois catégories d'informations complémentaires dont la mise en ouvre apparaît primordiale à la définition des activités du sacré comme à la perception des liens dynamiques qui les unissent à leur milieu d'origine. 


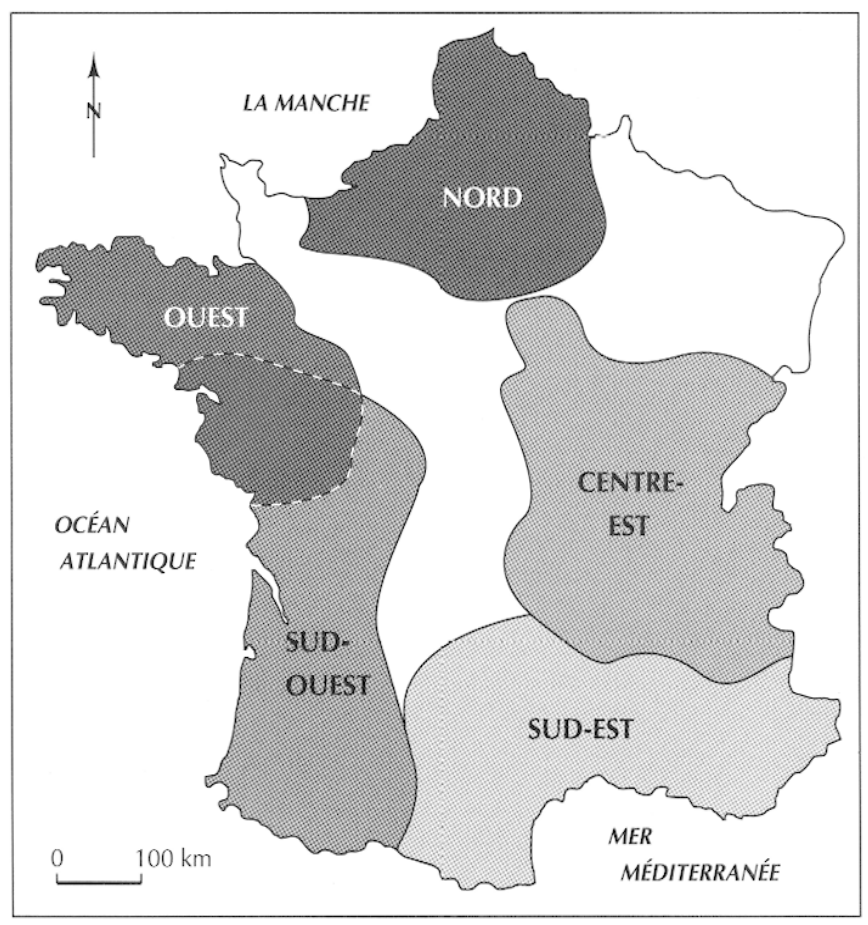

Fig. 1 - Carte des cinq grandes régions de la Gaule celtique concernées par les analyses du dossier (on se reportera pour le détail des sites aux cartes spécifiques en tête de chacun des chapitres).

- C'est en premier lieu l'indispensable synthèse des données factuelles récentes issues des fouilles, prospections et découvertes fortuites, en association avec les acquis antérieurs. Dans ce domaine, la part de la recherche programmée demeure forte, mais plusieurs sites majeurs ont été reconnus à l'occasion d'actions préventives. Surtout, l'intervention de chercheurs spécialisés dans l'anthropologie, la zoologie ou la carpologie, par exemple, est devenue un facteur déterminant dans l'amplification qualitative des approches cultuelles et de leur déroulement concret, des rituels probables de la commensalité aux offrandes déposées pour les divinités. De cette manière est remis en cause la seule notion de "sanctuaire " comme lieu sacrificiel, au profit des " enclos à banquets " dont les fossés regorgent sans doute davantage des reliquats de repas (même ritualisés !) que d'offrandes strictement dédiées aux divinités.

On commence par ailleurs à mieux percevoir certaines des modalités de l'insertion des activités profanes dans l'aire du sacré, tels les lieux de réunion ou les ateliers de frappes monétaires, et plus généralement de l'imbrication en habitats des aires consacrées, à des titres divers, au sein des activités du quotidien. De même, si la notion de sacrifices humains est une réalité qui n'est plus discutée (même si cette pratique demeure exceptionnelle), toute découverte de restes humains ne saurait forcément s'y rattacher. On préfère désormais parler de manipulation d'éléments du corps humain, en séparant avec discernement critique les vestiges de sacrifiés (dans des sanctuaires) de ceux liés aux rites de victoire (exposition de corps en trophées ou de têtes prélevées). Ainsi, la multiplication des découvertes de corps ou éléments de ceux-ci à proximité des habitats, généralement enfouis en silos abandonnés, laisse entrevoir la probabilité, par-delà le principe avancé de la relégation, de récupération de restes déjà partiellement décomposés et déposés à des fins propitiatoires pour les autres silos proches, toujours en activité. Parallèlement, l'idée de lieux destinés à la décomposition en milieu ouvert de " héros" morts au combat est avancée à propos du second enclos ovalisé de Ribemont-sur-Ancre (Somme). Très perceptible dans le Midi gaulois, la récupération dans l'habitat des restes (ici incinérés) de personnages héroïsés participe de la protection du site fortifié. Par ailleurs la tête humaine de personnages considérés comme majeurs peut être également conservée et nettoyée (après exposition des restes en plein air ou avant incinération); dans la basse vallée du Rhône, elle est présentée comme relique sous un masque réaliste, au sein de portiques à motifs symboliques peints. Les corps assis en fosse d'Acy-Romance montrent enfin l'étendue des rituels possibles en ce domaine et celle de nos méconnaissances actuelles.

- La deuxième démarche informative est celle de la relecture critique du fonds ancien des données, confronté aux connaissances contemporaines. La recherche archéologique sur le monde celtique souffre fondamentalement de l'absence d'une information conséquente de nature iconographique (le caractère iconoclaste des Celtes jusqu'à la conquête romaine) ou épigraphique (une forte tradition de l'oralité et d'un enseignement socioreligieux réservé essentiellement à une classe dirigeante). Dans le domaine des pratiques religieuses, la démarche pâtit également de l'absence d'une nécessaire terminologie commune recouvrant une claire définition de la nature des lieux concernés. Beaucoup de découvertes isolées ou bouleversées par la présence d'une édification gallo-romaine sont utilisées, souvent faute de mieux, parfois même sans approche taphonomique, gage élémentaire de leur valeur heuristique, pour avancer l'existence d'un espace cultuel au cours de l'âge du Fer. De même, récemment en région méditerranéenne, l'attribution votive de certains dépôts en grotte a été contestée au profit de simples cachettes à finalité plus profane. Parce que seul le sanctuaire, la forme monumentalisée d'un espace cultuel, paraît avoir bénéficié d'un réel effort de clarification, beaucoup d'autres lieux moins lisibles ou plus polyvalents sont encore crédités de cette appellation commode mais parfois trompeuse. La caractéri- 
sation d'un sanctuaire passe désormais par la reconnaissance d'ćlćmcnts qui lui sont consubstantiels, l'enceinte matérialisée, la structure sacrificielle, l'émanation divine (c'est souvent le bois sacré mais on peut imaginer d'autres formes naturelles, un rocher, un ancien mégalithe, une source, etc.). Il apparaît fondamental de reconnaître les attributs essentiels du lieu de culte pour le différencier d'autres à vocation plus politique que religieuse.

Nous avons précédemment souligné la nécessité de mieux caractériser sur le plan des rites et des cultes les vestiges entreposés dans les fossés de nombreux enclos gaulois. De même, les puits de la région toulousaine et leurs contenus donnent lieu depuis plusieurs décennies à des interprétations variées, voire contradictoires. Pour unc part d'cntrc cux au moins, unc nouvcllc analyse des étapes de leur abandon laisse entrevoir une utilisation initiale profane, écartant du même coup leur dévolution funéraire ou cultuelle. Le dossier montre également tout l'intérêt d'une considération plus critique de l'iconographie au travers de ses détails figurés (armement, bijouterie). Cet exercice, réalisé dans les régions méridionales, conduit à une meilleure distribution chronologique des sculptures et, du même coup, au témoignage précoce des cultes héroïques dès l'amorce de l'âge du Fer, en parallèle avec les exemples du sud de l'Allemagne et de l'Italie centrale.

- Enfin, toutes ces approches archéologiques des lieux et pratiques cultuelles intègrent les témoignages de l'Antiquité sur la Gaule en ce domaine, tout en respectant par une lecture critique la validité de leur complémentarité informative. La confrontation avec les textes anciens sur le culte, ses lieux et ses formes chez les Celtes, nous invite à une certaine humilité. Dans bien des cas, on aurait tendance à croire que l'archéologie ne découvre que ce que nous savions déjà ou que nous aurions dû savoir par la lecture de ces relations antiques. Bien sûr, cette boutade porte sa part de vérité, affirmation elle-même rendue possible par l'analyse des travaux de l'archéologie! Outre de donner forme et consistance à des textes souvent trop allusifs, les apports de terrain récents permettent de fixer dans le temps et dans l'espace les informations d'origine littéraire. Ainsi est-il édifiant de constater que les données ethnographiques de Poseidonios d'Apamée trouvent une correspondance matérielle en Gaule septentrionale pour une date très haute (fin du $\mathrm{IV}^{\mathrm{e}}$ et début du $\mathrm{III}^{\mathrm{e}}$ s. avant J.-C.) et non, comme on l'a cru longtemps, dans le seul arrière-pays de Marseille à la fin du II $^{\mathrm{e}} \mathrm{s}$. II ne fait guère de doute que la recherche future permettra également de comprendre bien des «bizarreries " et des anachronismes qui figurent dans la description des Gaulois au sein de l'œuvre de César.

\section{RÉALITÉ DES CROYANCES ET LISIBILITÉ DE LEURS PRATIQUES}

Les croyances des Gaulois, quelques-unes tout au moins, ne nous apparaissent plus tout à fait hors de portée. Mieux, la lecture de l'information archéologique des lieux de culte en regard des autres connaissances sur les sociétés contemporaines génère un renouvellement des problématiques culturelles. Mais en interne, au-delà des typologies commentées d'objets déposés dans les sanctuaires et des choix sacrificiels pratiqués, l'archéologie n'apporte qu'un concours incomplet et peu satisfaisant à une meilleure connaissance des rites pratiqués. On serait tenté de dire que la matière se trouve en priorité dans les textes antiques, les vestiges matériels n'étant là que pour accréditer ces récits lointains auxquels on ne savait quelle confiance accorder ! Si tel est le cas, cette contribution est déjà en soi remarquable, car elle permet de tirer le meilleur parti de sources d'autant plus précieuses qu'elles sont en nombre limité et ne peuvent, de ce fait, se conforter les unes les autres.

La validation archéologique des informations antiques est un des apports majeurs de la recherche récente sur les cultes à l'âge du Fer. Elle paraissait jadis relativement anecdotique. Elle montre aujourd'hui que les historiens et géographes grecs et latins ont puisé dans un nombre limité de sources, souvent de bonne qualité, s'appuyant toujours sur des faits authentiques, même s'ils furent souvent mal compris ou interprétés avec outrance par eux. Ainsi les temene de Poseidonios (chez Diodore), les luci de Pline et de Lucain ont trouvé une réalité. La découpe systématique du crâne de l'ennemi a été vérifiée. Son exposition sur les porches des habitats ou des sanctuaires aussi. Les progrès les plus remarquables concernent les coutumes liées au funéraire et aux croyances eschatologiques. La récente. découverte de Ribemont-sur-Ancre confirmerait les propos de Silius Italicus, d'Élien et de Pausanias sur la meilleure manière pour les héros de gagner un ailleurs bienheureux.

Mais surtout, la recherche archéologique récente montre que les croyances des Celtes étaient beaucoup plus diversifiées qu'on l'imaginait il y a une vingtaine d'années. Dans cette diversité où la part du traitement des corps reflète des conditions humaines et des destins post mortem variés, les funérailles solennelles décrites par César ou les étranges sépultures d'Acy-Romance devraient trouver leur place. 
Nota bene (petit rappel d'un phasage chronologique moyen pour l'âge du Fer gaulois) :

Hallstatt B2-B3 : vers 930-800

Hallstatt $C$ : vers $800-650$

Hallstatt D1 : vers $650-540$

Hallstatt D2 : vers $540-510$

Hallstatt D3 : vers $510-450$

La Tène A : vers $450-390$

La Tène Bl : vers $390-320$
La Tène B2 : vers $320-280$

La Tène Cl : vers 280-190

La Tène C2 : vers 190-140

La Tène D1a : vers $140-120$

La Tène Dlb : vers 120-80

La Tène D2a : vers 80-50

La Tène D2b : vers 50-30 